\title{
«Patentad, patentad!»:
}

\section{apuntes sobre la apropiación}

del trabajo científico por las grandes corporaciones multinacionales

\section{RAÚL DELGADO WISE* MÓNICA CHÁVEZ ELORZA**}

Asistimos a una época en la que las patentes se han convertido en un aspecto consustancial a la lógica de reestructuración neoliberal promovida por las grandes corporaciones multinacionales y los gobiernos imperialistas encabezados por Estados Unidos. La expansión, la concentración y la apropiación privada de los productos del general intelect han alcanzado ritmos hasta hace poco inimaginables y que atraviesan todos los poros de la sociedad capitalista, pero que lejos de favorecer una ruta progresista de desarrollo de las fuerzas productivas, conducen a una fase regresiva, oscurantista, en el avance del conocimiento, que profundiza el desarrollo desigual y atenta contra la naturaleza y la humanidad, a grado tal de convertirse en una amenaza de dimensiones y alcances civilizatorios.

* Docente investigador de la Unidad Académica de Estudios del Desarrollo, Universidad Autónoma de Zacatecas.

** Docente investigadora de la Unidad Académica de Estudios del Desarrollo, Universidad Autónoma de Zacatecas.
A lo largo de las últimas dos décadas y media se han producido drásticas transformaciones en los modos de generación y apropiación del conocimiento. Entre otras, las dinámicas de apropiación del trabajo científico y tecnológico mediante el patentamiento se han expandido y crecido a ritmos sin parangón en la historia del capitalismo contemporáneo. Se trata, en el fondo, de un viraje trascendental en la forma de operación y desarrollo de los sistemas de innovación, con epicentro en Estados Unidos, cuyos principales artífices son las grandes corporaciones multinacionales (CMN). Gran parte de la lógica que subyace en los acuerdos de libre comercio en boga, como el Tratado de Libre Comercio de América del Norte (TLCAN), la Asociación de Naciones del Sudeste Asiático (ASEAN, por sus siglas en inglés) y el Acuerdo Transatlántico de Cooperación Económica (TTP, por sus siglas en inglés), se apoya en este proceso y en el tejido institucional de raigambre neoliberal que lo sustenta, impulsado por el Banco Mundial (BM), el Fondo Monetario Internacional (FMI), la Organización Mundial del Comercio (OMC) y, recientemente, la Organización Mundial de la Propiedad Intelectual (WIPO, por sus siglas en inglés). 


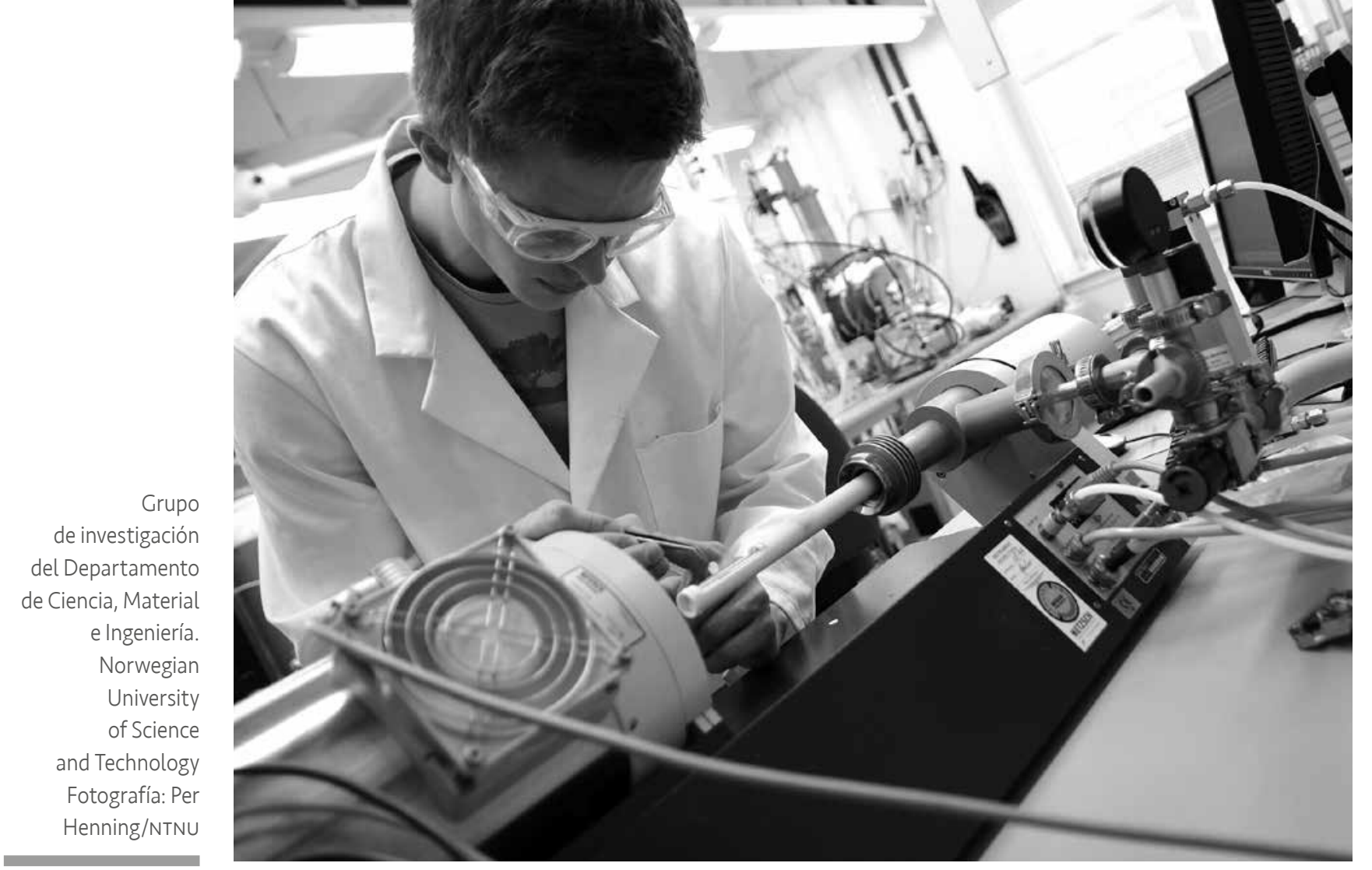

En el corazón de nuestro argumento subyace la hipótesis de que los sistemas de innovación atraviesan por una profunda fase de reestructuración comandada por las grandes corporaciones multinacionales, principalmente de origen estadounidense - asentadas en Silicon Valley y sus satélites-, la cual da lugar a una desbordante carrera por la generación y concentración de patentes. Bajo ese nuevo andamiaje, al lema «Acumulad, acumulad», el capital monopolista agrega la consigna « $\mathrm{Pa}-$ tentad, patentad», y en su insaciable afán de lucro torna el carácter progresista que Marx atribuyó al desarrollo de las fuerzas productivas de la sociedad en los primeros pasos del capitalismo en su contrario: una senda que atenta contra la naturaleza y contra la vida. Ello no sólo cuestiona la noción de desarrollo, también promueve la generación de nuevas formas de intercambio desigual que acentúan las asimetrías entre países, agudizan las desigualdades sociales, al tiempo que desajustan los mecanismos del sistema y lo sumergen en una profunda crisis que amenaza a la humanidad entera, de la que en apariencia no ha po- dido encontrar sino falsas salidas, como es el caso de la financiarización, el extractivismo y la superexplotación laboral.

\section{Reestructuración de los sistemas de innovación}

Las formas tradicionales de comprender los sistemas de innovación, como la triple hélice o el triángulo de Sábato, resultan anacrónicas para entender las nuevas dinámicas de «desarrollo» científico y tecnológico. Referirse al Sistema Nacional de Innovación es igualmente inadecuado para captar la complejidad de los ecosistemas dominantes. Al respecto, tomando como ejemplo el caso paradigmático de Silicon Valley -y sus satélites-, es posible distinguir cuatro ejes que a grandes pinceladas describen las dinámicas de generación y apropiación del trabajo científico-tecnológico que surgen bajo la égida de la globalización neoliberal: ${ }^{1}$

${ }^{1}$ Raúl Delgado Wise, «Unraveling mexican highly-skilled migration in the context of neoliberal globalization», in S. Castles, M. Arias Cubas \& D. Ozkul, D. (eds.), Social Transformation 
1. Internacionalización y fragmentación de las actividades de investigación y desarrollo en alusión a la preeminencia de modalidades «colectivas»: peer-to-peer, share economy, commons economy y crowdsourcing economy de innovación, a través de lo que se conoce como innovación abierta (open innovation). Se trata de modalidades de invención «extramuros», fuera del entorno de las CMN, que entrañan una apertura y redistribución espacial de actividades intensivas en conocimiento, con la creciente participación de socios externos - proveedores, clientes, subcontratistas, universidades y centros de investigación -, lo que ha dado paso a la permanente configuración y reconfiguración de redes de innovación.

2. Ciudades científicas, por medio de las cuales se producen sinergias colectivas que conducen a una aceleración de la investigación y el desarrollo (Silicon Valley, Silicon Valley periféricos o maquiladoras científicas), y en consecuencia de la innovación. En el fondo, como lo destaca AnnaLee Saxenian, ${ }^{2}$ es un nuevo paradigma que se aparta de los viejos modelos «cerrados» de investigación y desarrollo, y que abre el camino hacia una nueva cultura de la innovación basada en la flexibilidad, la descentralización y la incorporación, con diferentes modalidades, de nuevos y cada vez más numerosos jugadores que interaccionan en espacios locales y transnacionales (maquiladoras científicas).

3. Nuevas formas de control de las agendas de investigación y de apropiación de los productos del trabajo científico, es decir, las patentes, por las grandes CMN, a través de for-

and Migration: National and Local Experiences in South Korea, Turkey, Mexico and Australia, United Kingdom, Palgrave MacMillan, 2015, pp. 201-218.

2 Véase AnnaLee Saxenian, Local and Global Networks of Immigrant Professionals in Silicon Valley, San Francisco, Public Policy Institute of California, 2002; AnnaLee Saxenian, The New Argonauts: Regional Advantage in a Global Economy, Boston, Harvard University Press, 2006. mas multivariadas de subcontratación, asociación, manejo y diversificación del capital de riesgo y, lo más importante, la disposición de equipos especializados de abogados que negocian con los potenciales subcontratistas y operan como una suerte de head hunters, pues conocen los entretelones de la operación de los nuevos sistemas de patentamiento, como los establecidos mediante la WIPO. A las nuevas y complejas formas de gestión y control se les conoce como strategic investment. ${ }^{3}$

4. Expansión en el horizonte Norte-Sur de la fuerza de trabajo en áreas de Ciencia, Tecnología, Innovación y Matemáticas y el creciente reclutamiento de fuerza de trabajo altamente calificada proveniente de las periferias vía outsourcing (subcontratación) y offshoring (deslocalización). La migración altamente calificada desempeña un papel cada vez más relevante en los procesos de innovación, de modo que se promueve una paradójica y contradictoria dependencia del Norte respecto del Sur: cada vez más los generadores de patentes son originarios de los países periféricos; al grado que, de acuerdo con datos de la WIPO, en 2014 cerca de la mitad provenía de esas latitudes, pese a que alrededor de 75 por ciento de las patentes fue concentrado y apropiado por corporaciones multinacionales.

Con base en el nuevo escenario, que perfila lo que podría concebirse como la implantación de un Sistema Imperial de Innovación, a continuación explicaremos la estrategia en materia de derechos de propiedad intelectual seguida por Estados Unidos a partir de 1990. No debe perderse de vista - atendiendo a lo reseñado en este apartado- que las patentes encierran una permanente y paulatina tensión entre, por una parte, los beneficios de las grandes corporaciones y los intereses de los innovadores y, por otra parte, las necesidades de los consumidores y de la sociedad en general. En

\footnotetext{
${ }^{3}$ Titus Galama and Josek James, US Competitiveness in Science and Technology, Santa Mónica, RAND Corporation, 2008.
} 


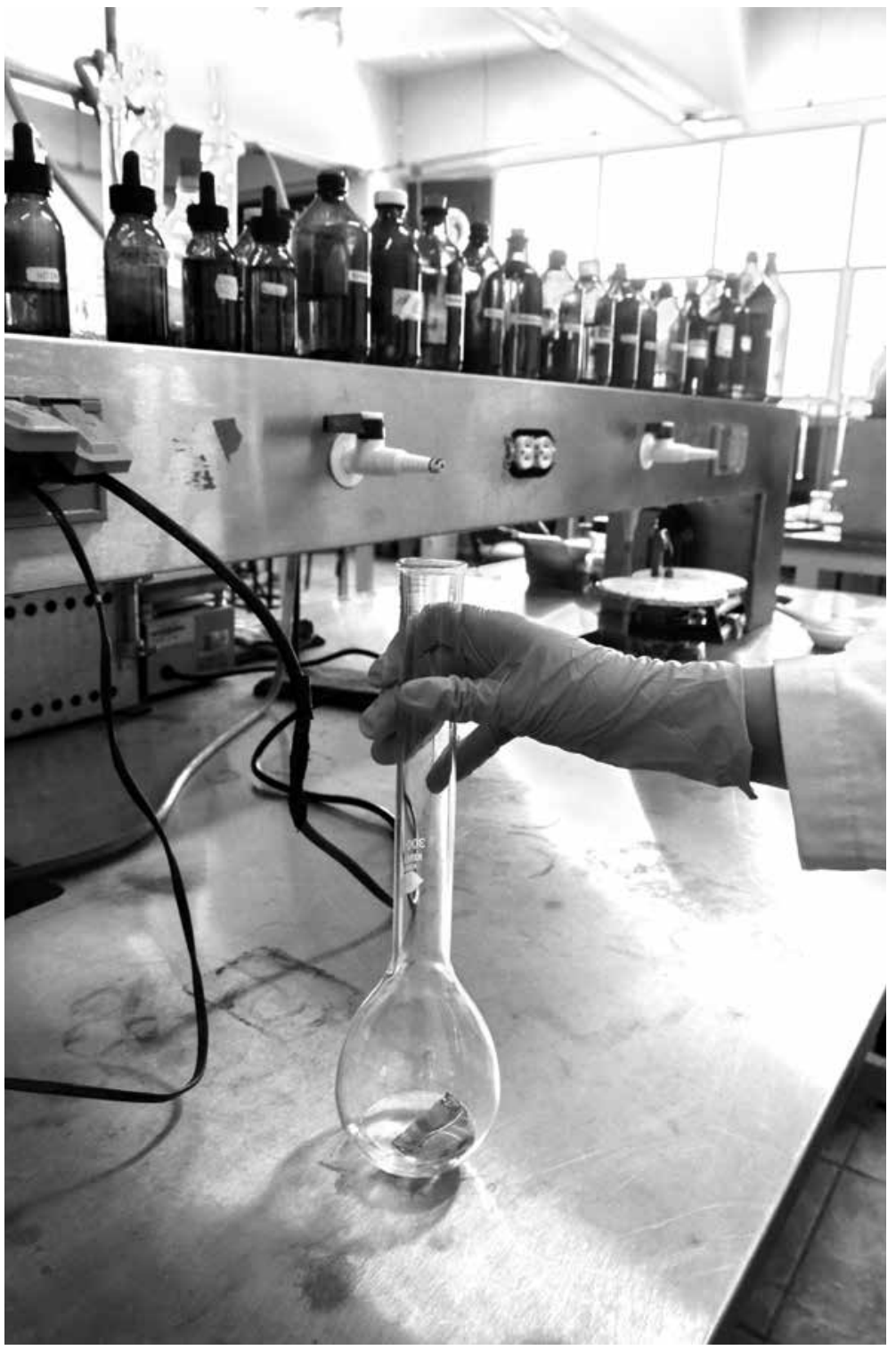

La innovación abierta (open innovation) es una modalidad

de invención «extramuros»,

fuera del entorno de las corporaciones multinacionales con la creciente participación de socios externos. Fotografía: Jorge Vázquez

Desde finales de la década de 1980 inicia en Estados Unidos una tendencia a legislar ad hoc, de conformidad con los intereses estratégicos de las grandes corporaciones, en materia de propiedad intelectual. ${ }^{4}$ Mediante las regulaciones impulsadas por la Organización Mundial del Comercio (OMC) - Acuerdo sobre los Aspectos de los Derechos de Propiedad Intelectual relacionados con el Comercio $-{ }^{5}$ dicha normatividad se expande a escala global a través de las negociaciones para la suscripción y puesta en marcha de los Tratados de Libre Comercio (TLC). ${ }^{6}$ Estas negociaciones se han realizado a través de la Oficina del Representante Comercial de Estados Unidos, quien a su vez ha protegido y representado los intereses de las industrias intensivas en el uso de la propiedad intelectual y farmacéutica - Business Software Alliance (BSA) en derechos de autor y Pharmaceutical Research and Manufacturers of America (PhRMA) en patentes farmacéuticas. ${ }^{7}$ Por su carácter multilateral, las disputas en materia de propiedad intelectual al seno de la OMC tienden a complejizarse, por lo que la estrategia estadounidense contempla la negociación bilateral del TLC con objeto de controlar mercados y acrecentar ganancias corporativas.

Un aspecto medular de los TLC ha sido inclinar la balanza de los procesos de intercambio comercial a favor de los inversionistas (las grandes corporaciones multinacionales) mediante la extensión y ampliación de la cobertura de las

${ }^{4}$ Peter J. Messitte, «Desarrollo del derecho de patentes estadounidense en el siglo XXI. Implicaciones para la industria farmacéutica», en Arturo Oropeza García y Víctor Manuel Guízar López (coords.), Los retos de la industria farmacéutica en el siglo XXI. Una visión comparada sobre su régimen de propiedad intelectual, Universidad Nacional Autónoma de México/Comisión Federal para la Protección contra Riesgos Sanitarios, 2012.

${ }^{5}$ Ibid., pp. 179-200.

${ }^{6}$ Víctor C. García Moreno, «El capítulo XVII del TLCAN y su influencia en la nueva Ley Mexicana del Derecho de Autor», en Manuel Becerra Ramírez (comp.), Estudios de derecho intelectual en homenaje al profesor David Rangel Medina, México, Universidad Nacional Autónoma de México, 1998, pp. 103-116.

${ }^{7}$ Estas industrias enfrentan altos costos de investigación y desarrollo (I\&D) mientras que el costo marginal de reproducción de los nuevos bienes es muy reducido; las patentes les proveen de una ventaja competitiva de mercado. Álvaro Díaz, op. cit. 
patentes y la flexibilización de los requisitos de patentamiento. Esto deja de lado las funciones que, en principio, debieran cumplir las propias patentes, tales como asegurar que la creación cuente con los requisitos de invención, originalidad y aplicabilidad socioeconómica, de igual modo que contribuya a difundir el conocimiento incorporado en la invención y evitar con ello la duplicación de esfuerzos. ${ }^{8}$ En otras palabras, los TLC atentan contra el fundamento del bien común que debiera tener el conocimiento, en tanto motor del progreso y desarrollo de las fuerzas productivas de la sociedad.

\section{Impactos de los acuerdos comerciales}

Uno de los impactos más importantes -y no tan obvio - de los TLC ha sido la adhesión de países periféricos a los convenios relacionados con patentes, ${ }^{9}$ así como la adecuación interna

${ }^{8}$ Idem.

${ }^{9}$ Parte de la agenda del TPP a través del convenio bilateral Estados Unidos-Unión Europea es penetrar en los mercados internos de algunos países de la UE, donde la penetración de las grandes corporaciones multinacionales ha sido en buena medida contenida mediante normas internas de protección. de sus leyes sobre propiedad intelectual. Ello ha ocurrido sin que tales adecuaciones guarden relación alguna con el nivel interno de desarrollo de la ciencia y la tecnología. Como afirma Shadlen: «México adoptó un sistema de patentes que es apropiado para un país con infraestructura científica, tecnológica e industrial significativamente más avanzada».10 Lo cual cobra mayor sentido si se considera que la generación de patentes se vincula con el gasto en investigación y desarrollo, el tipo de inversión que se lleva a cabo en investigación científica aplicada o innovación patentable, así como con la capacidad de absorción de conocimientos tecnológicos. ${ }^{11}$ A contra sensu, si no operara la lógica corporativa e imperialista referida, la legislación sobre propiedad intelectual debiera ser diametralmente distinta, lo que permitiría

${ }^{10}$ Kenneth C. Shadlen, «Propiedad intelectual para el desarrollo en México», en Kevin P. Gallagher, Enrique Dussel Peters y Timothy A. Wise (eds.), El futuro de la política de comercio en América del Norte: lecciones del TLCAN, Pardee Center Task Force Report/Universidad Autónoma de Zacatecas/Global Development and Environment Institute/Boston University Frederick S. Pardee Center/Universidad Nacional Autónoma de México, 2011, p. 68, http://www.ase.tufts.edu/gdae/policy_ research/pardee.html

${ }^{11}$ Álvaro Díaz, op. cit.

Gráfica 1. Evolución de las patentes registradas en EUA

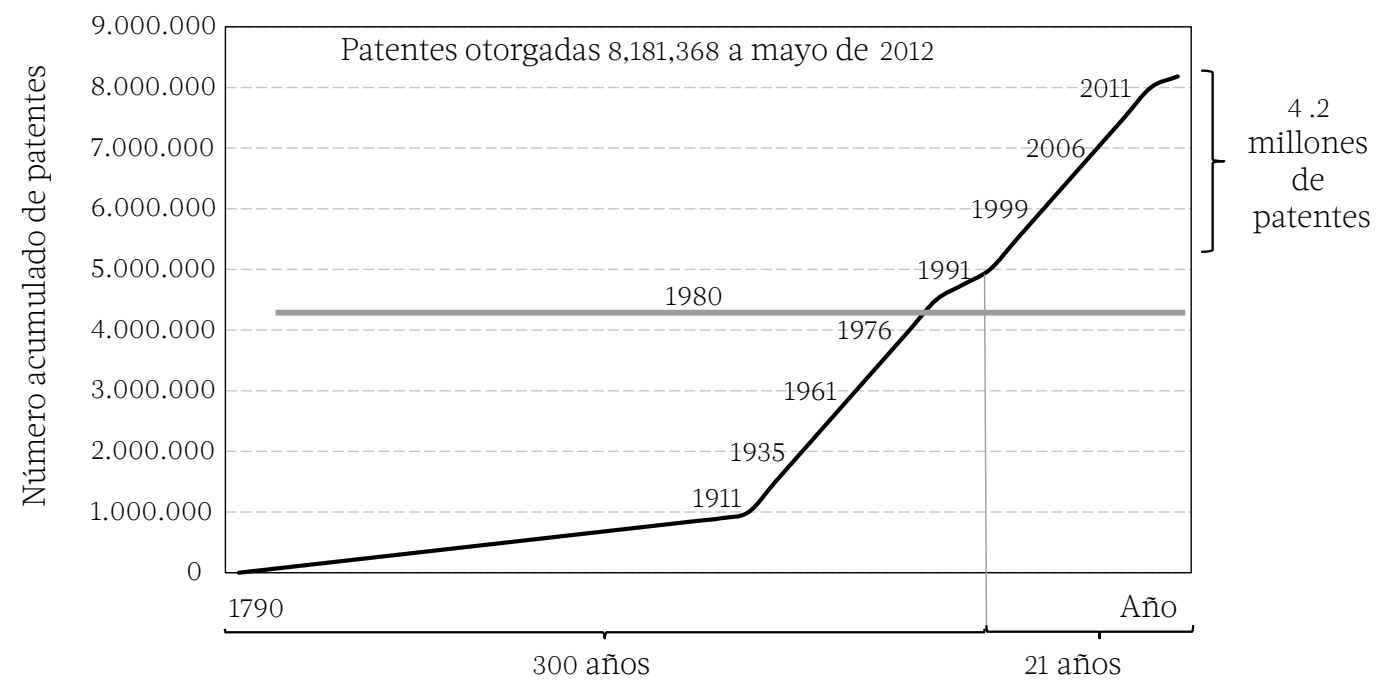

Fuente: Gina Smith, «Can the U.S. patent system be saved?», ComputerWorld, 2012,

http://www.computerworld.com/article/2505817/it-management/can-the-u-s-patent-system-be-saved-.html 
Cuadro 1. Solicitudes de patentes hechas por migrantes, 1990, 2000 y 2010

\begin{tabular}{|c|c|c|c|c|c|c|c|}
\hline \multirow{2}{*}{ Dirección de flujos } & \multirow{2}{*}{$\begin{array}{l}\text { Periodo } \\
1990-2010\end{array}$} & \multicolumn{2}{|c|}{ Año } & \multirow{2}{*}{$\begin{array}{c}\text { Tasa de } \\
\text { crecimiento } \\
\text { 1990-2010 }\end{array}$} & \multicolumn{3}{|c|}{ Distribución porcentual } \\
\hline & & 1990 & 2010 & & $1990-2010$ & 1990 & 2010 \\
\hline Total & 820,072 & 2,922 & 91,720 & 18.8 & 100.0 & 100.0 & 100.0 \\
\hline \multicolumn{8}{|l|}{ Sur-Norte } \\
\hline Países no OCDE a países OCDE* & 317,946 & 654 & 39,936 & 22.8 & 38.8 & 22.4 & 43.5 \\
\hline \multicolumn{8}{|l|}{ Norte-Sur } \\
\hline Países OCDE a países no OCDE* & 23,598 & 54 & 3,822 & 23.7 & 2.9 & 1.8 & 4.2 \\
\hline \multicolumn{8}{|l|}{ Norte-Norte } \\
\hline Países OCDE a países OCDE* & 464,900 & 2,208 & 45,880 & 16.4 & 56.7 & 75.6 & 50.0 \\
\hline \multicolumn{8}{|l|}{ Sur-Sur } \\
\hline Países no OCDE a países no OCDE* & 13,628 & 6 & 2,082 & 34.0 & 1.7 & 0.2 & 2.3 \\
\hline
\end{tabular}

Patent Cooperation Treaty (PCT), World International Patent Organization (WIPO).

*Países de la OCDE, no incluye a México, Chile ni Turquía.

Fuente: Estimaciones propias con datos de E. Miguelez y C. Fink, «Measuring the international mobility of inventors: a new database», WIPO Economic Research Working Paper No. 8, 2012, y países firmantes del PCT, WIPO, http://www.wipo.int/ pct/en/pct_contracting_states.html

a los países periféricos desarrollar sus capacidades endógenas en materia de ciencia, tecnología e innovación.

Adicionalmente, entre 1991 y 2011 se genera una dinámica desbordante de crecimiento en el plano del patentamiento que se sintetiza en el lema antes referido y, por lo demás, ajeno a una visión positiva y equitativa del progreso: patentad, patentad. En efecto, como se aprecia en la gráfica 1, en las dos décadas referidas se crearon en Estados Unidos más patentes que en 300 años de historia previa. El desproporcionado y a todas luces espectacular crecimiento, aunque se relaciona con la lógica de la reestructuración de los sistemas de innovación descrita en el primer apartado, entraña también una cierta ampliación, vulgarización y aprisionamiento (enclosure) de lo que materialmente tiene sentido patentar y que atenta contra la lógica del conocimiento como bien común, en tanto producto del general intelect, conforme a la definición de Marx sobre el trabajo científico.

Más aún, en ocasiones se trata de patentes adquiridas para que no se apliquen o su aplicación se postergue con el afán propio del capital monopolista de controlar y regular los merca- dos; ello da lugar a lo que Guillermo Foladori concibe como «ciencia ficticia», por su carácter especulativo, que se asemeja a la noción acuñada por Marx de capital ficticio. ${ }^{12}$

Según se desprende del cuadro 1, Estados Unidos despunta como la primera potencia capitalista del mundo en materia de innovación: $:^{13}$ concentra 28 por ciento de la totalidad de patentes gestionadas. Al considerar al total de países de la OCDE - sin incluir a México, Chile y Turquía - se aprecia que, conjuntamente, concentran 90 por ciento de las solicitudes mundiales de patentes. Asimismo, se advierte un nivel extremadamente bajo de participación de América Latina y el Caribe en la generación de patentes, cuyo aporte al cúmulo global de solicitudes consignadas fue de apenas 0.7 por ciento.

Tras este aumento acelerado de las solicitudes de patentes se registra, en primer lugar, un

${ }^{12}$ Guillermo Fodalori, «Ciencia ficticia», Estudios Críticos del Desarrollo, vol. IV, núm. 7, 2014, pp. 41-66.

${ }^{13}$ En sintonía con su dominio en el plano de las patentes, Estados Unidos sigue siendo la primera potencia imperialista del planeta, pues domina también en el plano de las mayores corporaciones multinacionales, la moneda mundial, la fuerza militar y ejercer control sobre los principales organismos internacionales: BM, FMI, OMC y WIPO. 
Diagrama 1. Ganancias de los arreglos en disputas

Inversor-Estado (ISDS) (millones por caso adjudicado en paréntesis)

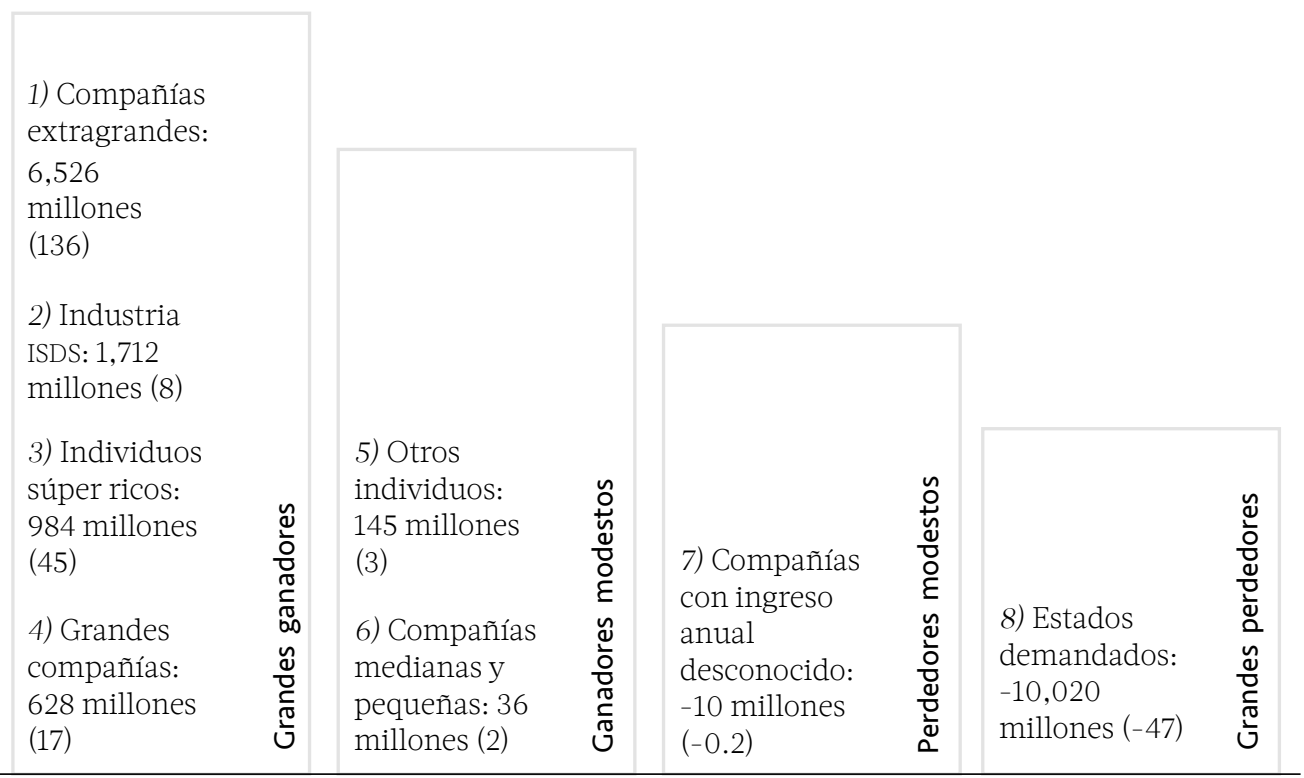

Notas: 1) Ingreso anual >10 billones USD, 2) Abogados y mediadores principalmente,

3) Riqueza neta >100 millones USD, 4) 10 billones USD >Ingreso anual >1 billón USD,

5) Riqueza neta <100millones USD, 6) y 7) 1 billón USD < Ingreso anual < 100 millones USD

Fuente: Gus Van Harten y Pavel Malysheuski, «Who has benefited financially from investment treaty arbitration? An evaluation of the size and wealth of claimants», Osgoode Legal Studies Research Paper No. 14, Vol. 12, Issue 3, 2016, pp. 12-13.

incremento de las solicitudes extranjeras en países periféricos. En el caso de México, con la entrada en vigor del TLCAN se da un proceso de sustitución de patentes nacionales por extranjeras. ${ }^{14}$ En segundo lugar, de manera un tanto paradójica, en la Oficina de Patentes y Marcas Registradas de Estados Unidos se aprecia una tendencia hacia el aumento de solicitudes de extranjeros, principalmente provenientes de países del Sur.

El tercer lugar engloba la industria farmacéutica, donde se presentan los efectos más visibles de las legislaciones aprobadas con relación a quiénes ganan y quiénes pierden. Los ganadores son las grandes corporaciones multinacionales, con ganancias provenientes de mercados monopólicos y de compensaciones por atrasos «injustificados» en la tramitación de

${ }^{14}$ Álvaro Díaz, op. cit. patentes y permisos de comercialización; mientras que los perdedores tienden a ser los consumidores finales, que no pueden adquirir los medicamentos a precios de monopolio impuestos, ni acceder, por efecto de los derechos de exclusividad encarnados en las patentes, a medicamentos genéricos. ${ }^{15} \mathrm{~A}$ esto último se agrega la llamada regla 90-10, que alude que 90 por ciento de la investigación se dirige a enfermedades que afectan a 10 por ciento de la población. ${ }^{16}$

En cuarto lugar, el arbitraje internacional en materia de propiedad intelectual ha resultado

\footnotetext{
${ }^{15}$ Alejandro Luna, «Patentes de invención. Patentes farmacéuticas, protección de datos clínicos y otros temas de interés para la industria farmacéutica en México», en Arturo Oropeza García y Víctor Manuel Guízar López, op. cit

${ }^{16}$ Stiglitz, 2010, citado en Julen Berasaluce Iza y Salvador F. Ruiz Medrano, «Patentes y otros incentivos a la innovación. Perspectiva económico-legal aplicada al caso de medicamentos», en Arturo Oropeza García y Víctor Manuel Guízar López, op. cit.
} 
ser un negocio bastante lucrativo para las grandes corporaciones y los bufetes de abogados esmandados $\mathrm{y}$, a final de cuentas, del ciudadano común. Van Harten y Malysheuski estiman que los montos agregados en transferencias de los estados demandados hacia los inversionistas fue de 9 mil 164 millones de dólares en 214 casos y 856 millones de dólares hacia la industria del arbitraje inversionista-Estado (véase diagrama 1).

Por último, y como efecto de las regulaciones internacionales referidas, muchos países se ven orillados a adherirse a las agendas de investigación en ciencia y tecnología que devienen en consecuencias nefastas para sus economías y sociedades en materia financiera, económica y medioambiental. Van Harten se refiere a este fenómeno como una suerte de compra de soberanía por parte de las grandes corporaciones multinacionales. ${ }^{17}$

\section{Conclusiones}

La reestructuración de los sistemas de innovación constituye un mirador privilegiado para analizar y comprender el significado e implicaciones de las formas de apropiación del conocimiento que distinguen a la globalización neoliberal y que subyacen a la lógica de dominación que acompaña a los tratados de libre comercio promovidos por las grandes corporaciones multinacionales y las principales potencias imperialistas encabezadas por Estados Unidos. pecializados, en detrimento de los gobiernos de-

No se trata de acuerdos en los que todos los participantes ganan sino de estrategias que profundizan las dinámicas de desarrollo desigual que caracterizan al capitalismo contemporáneo; pero no sólo eso, conducen también a una carrera desenfrenada hacia la expansión y apropiación de los productos del trabajo científico y tecnológico bajo una perspectiva de obtención de ingentes ganancias monopólicas a cualquier costo.

Todo indica, en este sentido, que nos encontramos ante una crisis, con visos de terminal, de la modernidad capitalista, que reclama avanzar hacia una modernidad alternativa; es decir, una modernidad no capitalista que

implique una verdadera abundancia y una verdadera emancipación (...) una modernidad que nunca fue, que nunca existió, no -como dice Habermas - completar el proyecto de modernidad, sino inventar otra distinta que fue posible incluso desde antes y que fue reprimida y negada, y hasta ahora postergada. ${ }^{18}$

En el fondo, se trata de avanzar hacia una ruta de transformación social radical, centrada en las necesidades sociales y en armonía con la naturaleza, que conlleve, entre otras cosas, la defensa a ultranza de los bienes comunes tangibles e intangibles. ${ }^{19}$

\footnotetext{
${ }^{18}$ Bolívar Echeverría, Antología. Crítica de la modernidad capitalista, La Paz, Oxfam/Vicepresidencia del Estado Plurinacional de Bolivia, 2011, p. 290.

${ }^{19}$ Cristian Laval y Pierre Dardot, Común: ensayo sobre la revolución en el siglo XXI, Barcelona, Gedisa, 2015.
}

${ }_{17}$ Van Harten, «Reformando el régimen de inversión del TLCAN», en Kevin P. Gallagher et al., op. cit. 